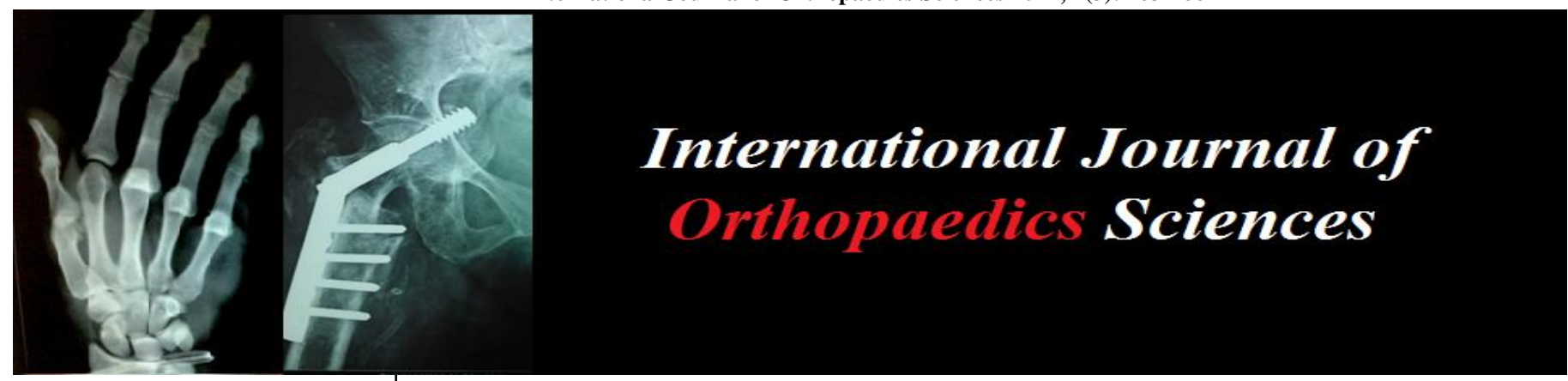

E-ISSN: 2395-1958

P-ISSN: 2706-6630

IJOS 2021; 7(3): 463-466

(C) $2021 \mathrm{IJOS}$

www.orthopaper.com

Received: 07-05-2021

Accepted: 11-06-2021

\section{Chetan Giroti}

DNB Orthopaedics. Govt Doon

Medical College, DehraDun,

Uttarakhand, India

\section{Akshat Mittal}

DNB Orthopedics. Govt. Doon

Medical College, Dehradun,

Uttarakhand, India

\section{Dhaval Gotecha}

MS Orthopedics, Govt. Doon

Medical College, Dehradun,

Uttarakhand, India
Corresponding Author: Akshat Mittal

DNB Orthopedics. Govt. Doon Medical College, Dehradun,

Uttarakhand, India

\section{Comparison of steroid injection and platelet-rich plasma injection in the treatment of De-Quervain Tenosynovitis}

\section{Chetan Giroti, Akshat Mittal and Dhaval Gotecha}

DOI: https://doi.org/10.22271/ortho.2021.v7.i3g.2789

\section{Abstract}

Aim: The aim of this study was to evaluate the therapeutic effect of corticosteroid and platelet-rich plasma (PRP) injections in the treatment of De Quervain Tenosynovitis.

Methods: The study group comprised of 49 patients with 50 hands divided into two groups. In the corticosteroid group (Group A; $\mathrm{n}=28), 1 \mathrm{ml}$ of methylprednisolone $(40 \mathrm{mg} / \mathrm{ml})$ was injected along with 1 $\mathrm{ml}$ of $0.5 \%$ bupivacaine. In the PRP group (Group B; $\mathrm{n}=22$ ), PRP was obtained from the patients' own blood and injected in a single dose. Patients were evaluated using Visual analogue scale (VAS), the shortened disabilities of the arm, shoulder and hand questionnaire (DASH) at baseline, one and three months after the injection.

Results: Overall, 44 females and 5 males were included with 28 hands in Group A and 22 hands in Group B. The CS group had better pain relief, hand function tests at 1 month and at 3 months follow-up, the PRP group had better pain relief, hand function tests than CS group.

Conclusion: PRP therapy proves to be more effective in relieving pain than corticosteroid injections in treatment of De-Quervain Tenosynovitis.

Keywords: DQVD, platelet-rich plasma, corticosteroid, wrist pain

\section{Introduction}

De Quervain's disease or entrapment tendinitis is defined as edematous stenosing tenosynovitis of the first dorsal compartment of the hand ${ }^{[1,2]}$. It is generally caused by abuse or an increase in repetitive activity, resultant in microtrauma from monotonous gliding of the first dorsal compartment tendons (abductor pollicis longus or APL, and extensor pollicis brevis or EPB) under the sheath of the first compartment above the styloid of the radius causing thickening of the extensor retinaculum of wrist ${ }^{[1,3]}$. De Quervain's typically present in the fifth and sixth decades of life, and common in pregnant and lactating females ${ }^{[4]}$.

Conservative therapy is the first line of treatment, including activity modifications, splinting, occupational or physical therapy, analgesic or nonsteroidal anti-inflammatory medications, and corticosteroid injection ${ }^{[5]}$. Up to $83 \%$ of patients report relief with a single corticosteroid injection ${ }^{[6]}$. Most common proposed theory is the chronic repetitive tendon overload ${ }^{[7,8]}$. Microscopic failure occurs within the tendon at high loads and eventually leads to alterations at the cellular level, which weaken the mechanical properties ${ }^{[8]}$. Tendon microtrauma can lead to individual fibril degeneration due to stress across the tendon, which can accumulate over time, leading to chronic tendinopathy ${ }^{[8]}$.

Platelet-rich plasma (PRP) injections that contains Growth factors are nowadays being used for tendinopathies as a recent strategy in the clinical practice to provide a regenerative stimulus for tendon healing ${ }^{[9]}$. PRP is a set of autologous platelet products used to reduce pain and speed up recovery from injury. Its basic role is to mimic and enhance the natural processes of healing by bringing to the injury site a set of molecules that will accelerate functional recovery, and even regenerate the tissue ${ }^{[10]}$.

The purpose of this study was to evaluate the comparison of therapeutic effect of corticosteroid and PRP injections in the treatment of De-Quervain Tenosynovitis. 


\section{Materials and Methods}

This retrospective study includes 50 hands of 49 patients with one patient of bilateral disease with symptoms and signs suggestive De-Quervain Tenosynovitis between Feb 2018 and June 2019 after a written consent from all patients. The Patients hands were divided into two groups, Group I (CS): Included 28 hands which received single CS injection with $1 \mathrm{ml}$ Methylprednisolone with $1 \mathrm{ml}$ of $0.5 \%$ bupivacaine. Three patients were males and 24 patients were females, their age ranged from 31 to 59 years. Group II (PRP): Included 22 hands which received PRP injection, 2 patients were males and 20 patients were females, their age ranged from 27-60 years. All the patients had symptoms non-responsive or recurrence of symptoms following conservative and physical therapy.

Patients below the age of 18 years, with previous history of fracture or surgery on the affected hand and wrist, those with previous history of steroid injections, infections or systemic diseases, Pregnancy, arthritis and patients on anti-platelet medication and oral steroids were excluded from the study. All patients were instructed to stop taking NSAIDs 3 weeks before the procedure.

\section{Procedure}

In Group A, a 22-gauge needle connected to a 5cc syringe containing $1 \mathrm{ml}$ of Methylprednisolone $(40 \mathrm{mg} / \mathrm{ml})$ with $1 \mathrm{ml}$ of $0.5 \%$ bupivacaine was prepared and injected into the tendon sheath. Light dressing was applied.

In Group B, $30 \mathrm{ml}$ of patients' blood was withdrawn and inserted into pre-packed PRP kits (Tricell) along with $5 \mathrm{ml}$ of anticoagulant $10 \%$ sodium citrate. The PRP sample was prepared by a double centrifugation process. The first centrifuge was done at $3200 \mathrm{rpm}$ for $4 \mathrm{mins}$. The cellular component was separated from the fluid component and a second centrifuge was them performed at $3300 \mathrm{rpm}$ for 3 mins. Following this, approximately 3-4 ml was obtained and injected into the tendon sheath. Patients were advised to avoid any physical activity for the next 48 hours and were allowed to ice the wrist joint and to take $\mathrm{N}$ on steroidal anti- inflammatory drugs.

Pre-procedure and on final follow-up, patients were assessed for their symptoms using Visual Analog Scale (VAS) and Disabilities of Arm, Shoulder and Hand (DASH) score. All variables of the DASH score were independently evaluated by an orthopedic junior resident. The researcher was blinded to the study purpose to avoid any bias in study treatment and/or failures.

\section{Results}

The present study included 50 hands in 49 patients divided into two groups. Group A received CS injection, and group B received PRP injection. The mean age of patients in Group A was 43.16 years and in Group B was 44.44 years. Group A had 3 males and 24 females, whereas Group B comprised of 2 males and 20 females, comprising a total of 5 males $(10.2 \%)$ and 44 females $(89.8 \%)$. The mean difference in Visual Analogue Scale (VAS) between the baseline evaluation and after 1 month of injection, there was more reduction in VAS score in group I. While there was more reduction in VAS score in group II as regards the mean difference between the results after 1 and 6 months of injection.

As regards the mean difference in Disabilities of the arm, shoulder and hand question are (DASH) between the baseline evaluation and after 1 month of injection, there was more improvement in DASH score in group I. While there was more improvement in DASH score in group II as regards the mean difference between the results after 1 and 6 months of injection.

None of the patients included in the study had complaints of any side effects after administering PRP. No infection or any other complications were reported at the end of 3 months. All patients in Group B showed steady decline in symptoms, 2 patients from Group A both females showed initial improvement, and then later on the symptoms showed recurrence, however not as debilitating as in pre-procedure records. Figures 1 and 2 compare the pre-procedure and postprocedure final follow up records of VAS and DASH scores.

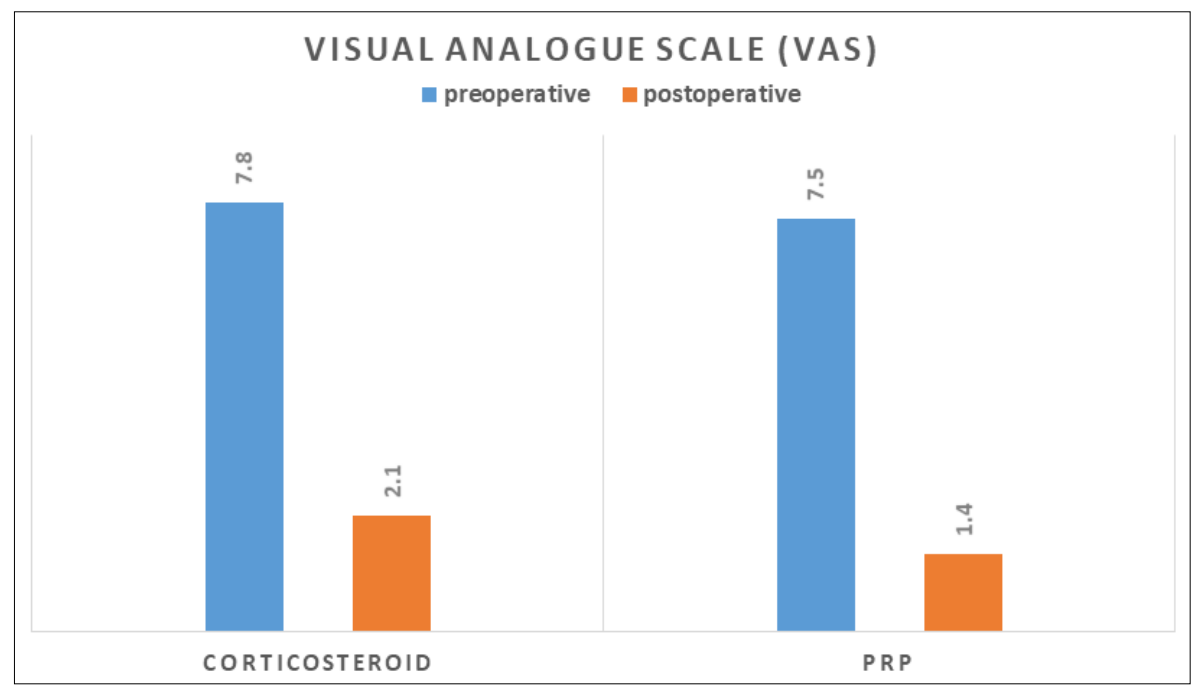

Fig 1: Comparison of pre-procedure and post-procedure VAS scores 


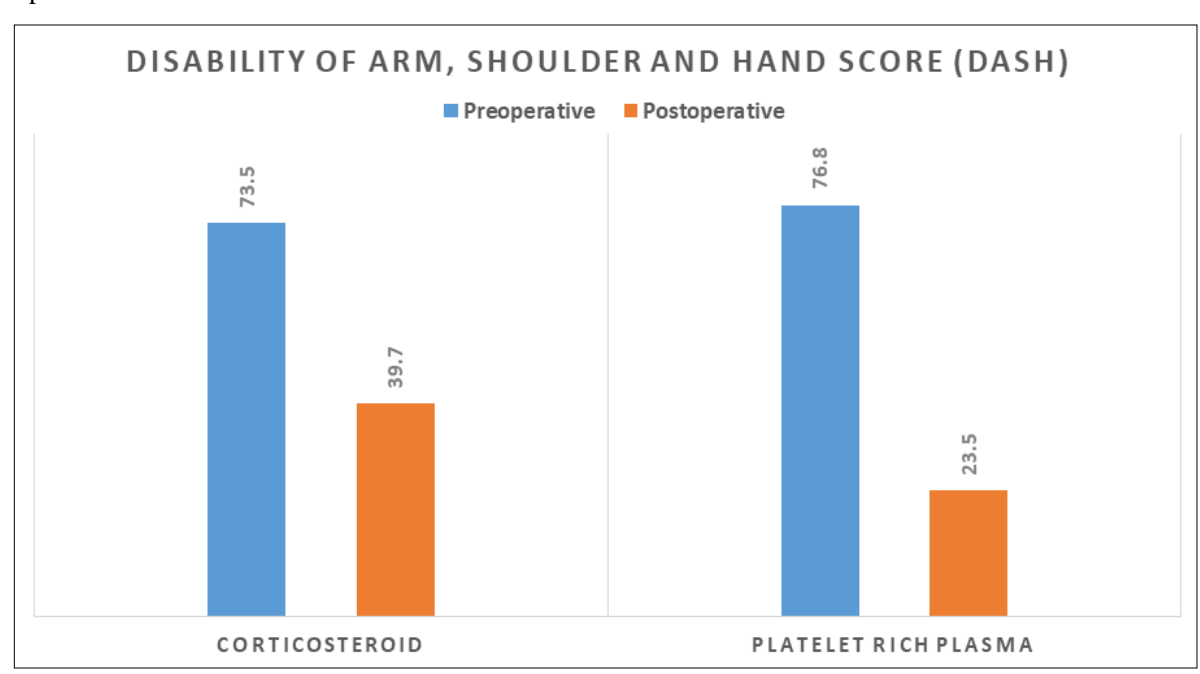

Fig 2: Comparison of pre-procedure and post-procedure DASH Score

\section{Discussion}

DQVD is more common in people who use their hands frequently as in cooking, piano playing, knitting, typing, working in the garden, playing golf, or lifting baby.

The present study aimed to compare the efficacy of corticosteroid versus platelet-rich plasma in treatment of DeQuervain Tenosynovitis. In present study we found a more improvement in Corticosteroid group than Prp group in regards to DASH score between the baseline evaluation and after 1 month of injection. While there was more improvement in DASH score in group II as regards the mean difference between the results after 1 and 6 months of injection. Showing a significant improvement in VAS and DASH scores in PRP group as compared to corticosteroid group in long term. Our results were consistent with other studies in literature.

A previous study found that the initial VAS score was 9.42 in the studied 141 patients with DQVD, and the mean postprocedural 6 months VAS was 3.92, a statistically significant improve-ment was observed in terms of pain relief after a single PRP injection in DQVD ${ }^{[11]}$. One more study included 30 patients who were injected once by PRP had reported decrease of the mean VAS from 5.9 to 2 after 6 months of follow-up, and increase in the pain free movement in all ranges without any significant complications ${ }^{[12]}$. A previous study on Lateral epicondylitis, is respect to mean DASH, they found at the baseline was (88 vs. 88) in PRP group and CS group respectively. After 1 month it was (62.5 vs. 53.13) in PRP and corticosteroid group respectively with lower score in CS group, after 3 months it was (34.16 vs. 44.33) with more reduction of score in the PRP group. This is in agreement with our study ${ }^{[13]}$.

It is the opinion in PRP that platelets after activation, release many growth factors, including platelet-derived growth factor, transforming growth factor, and epidermal growth factor, which may aid in tissue regeneration by angiogenesis, chemotaxis, cell proliferation ${ }^{[14]}$ thus stimulating a cascade to recruit reparative cells while also inhibiting apoptosis and metalloproteinase activity.

Our study had a few limitations. First, we did not have a control group. Second, we did not use ultrasound guidance to administer the injections, hence we were not aware of the preprocedure pathological condition of the extensor tendons of the first compartment. Third, with the use of PRP, we did not measure the pre-centrifuge and post-centrifuge platelet concentration in any of the samples, hence no standard dose of administration could be quantified. Another drawback of our study was the exclusion of patients previously treated with corticosteroids so we are not aware that corticosteroid refractory cases could be treated with corticosteroid or not.

\section{Conclusion}

So PRP injection in DQVD is considered a successful alternative treatment of healing effect, reducing pain with no side effects. We found that this treatment gives the maximum efficacy in the intermediate term, while CS has short term effect.

\section{References}

1. Retig AC. Athletic injuries of the wrist and hand. Part II: overuse injuries of the wrist and traumatic injuries to the hand. Am J Sports Med 2004;32(1):262-273.

2. Avci S, Yilmaz C, Sayli U. Comparison of nonsurgical treatment measures for de Quervain's disease of pregnancy and lactation. J Hand Surg 2002;27A(2):322324.

3. Dawson C, Mudgal CS. Staged description of the Finkelstein test. J Hand Surg 2010;35A(9):1513-1515.

4. Avci S, Yilmaz C, Sayli U. Comparison of nonsurgical treatment measures for de Quervain's disease of pregnancy and lactation. J Hand Surg 2002;27A(2):322324.

5. Ilyas AM, Ast M, Schaffer AA, Thoder J. de Quervain tenosynovitis of the wrist. J Am Acad Orthop Surg 2007; 15:757-764.

6. Richie CA, Briner WW. Corticosteroid injection for treatment of de Quervain's tenosynovitis: A pooled quantitative literature evaluation. J Am Board Fam Pract 2003;16:102-106.

7. Kannus P, Jozsa L, Natri A. Effects of training, immobilization and remobilization on tendons. Scand $\mathbf{J}$ Med Sci Sports 1997;7:67-71.

8. Rees JD, Maffulli N, Cook J. Management of tendinopathy. Am J Sports Med 2009;37:1855-1867.

9. Filardo G, DI Matteo B, Kon E, Merli G, Marcacci M. Platelet-rich plasma in tendon-related disorders: Results and indications. KneeSurg Sports Trau-matol. Arthrosc 2018;26:1984.

10. Anitua E, Prado R, Nurden AT, Nurden P. Characterization of Plasma Rich in Growth Factors (PRGF): Components and formulations. In: Anitua E, Cugat R, Sánchez M. (eds) Platelet Rich Plasma in Orthopaedics and Sports Medicine. Springer, Cham. Chapter II, 2018, 29-30. 
11. Jeyaraman M. The Prospective Study on Efficacy and Functional Outcome of Autologous Platelet Rich Plasma Injection in Musculoskeletal Disorders. EC Orthopaedics 2018;9:849-863.

12. Al-Ardi IM. Platelet-rich plasma as a treatment for DE Quervain's Disease. Al-Qadisiyah Medical Journal 2017;13(23):197-201.

13. Yadav R, Kothari SY, Borah D. Comparison of local injection of platelet rich plasma and corticosteroids in the treatment of lateral epicondylitis of humerus. Journal of clinical and diagnostic research: JCDR 2015, 9(7). RC05.

14. Rha DW, Park GY, Kim YK, Kim MT, Lee SC. Comparison of the therapeutic effects of ultrasoundguided platelet-rich plasma injection and dry needling in rotator cuff disease: A randomized controlled trial. Clin Rehabil 2013;27:113-122. 